\title{
Spontaneous internal carotid artery rupture during neck dissection in osteogenesis imperfecta patient
}

\author{
Giuditta Mannelli MD*, Alberto Deganello MD, Maria Rosa Laganà MD, Oreste Gallo MD \\ First Clinic of Otolaryngology, Department of Surgery and Translational Medicine, University of Florence, Via Largo Brambilla 3, 50134 Florence, Italy
}

\section{A R T I C L E I N F O}

Article history:

Received 4 April 2014

Accepted 24 May 2014

Available online $\mathrm{xxx}$

\section{Keywords:}

Connective tissue disorder

Osteogenesis imperfecta

Major cervical vessel rupture

Spontaneous rupture of ICA

Weakness of the vessel wall

\begin{abstract}
A B S T R A C T
Objective: Several connective tissue disorders are associated with vascular wall abnormalities, including spontaneous dissection of the cervical arteries. Osteogenesis imperfecta (OI) is a hereditary disorder, with rare neurovascular complications, which potentially lead to life-threatening events. Methods: We presented a case of spontaneous internal carotid artery (ICA) rupture that occurred in a 52year-old-woman, suffering from OI type I, and who underwent a bilateral modified radical neck dissection (mRND) plus total thyroidectomy for a T4aN1b thyroid cancer. During mRND, an ICA's spontaneous rupture occurred.

Results: Histopathologic report suggested a structural defect of the arterial wall without cancer infiltration. The patient did not experience any neurologic complications.

Discussion: Head and neck surgeons have to be aware about early clinical recognition of possible cervical vascular abnormalities, in patients with connective tissue disorders, potentially responsible for dramatic vascular rupture during cervical surgical procedures.
\end{abstract}

(c) 2014 Elsevier Ireland Ltd. All rights reserved.

\section{Introduction}

Many studies report the presence of structural alterations of vascular walls in patients suffering from connective tissue disorders such as Ehlers-Danlos syndrome (eDS), fibromuscular dysplasia, osteogenesis imperfecta (OI) and Marfan syndrome; these connective abnormalities are known to be risk factors for artery dissection or rupture [1]. Unfortunately, common imaging analyses (TC scan, MRI, Doppler sonography) could diagnose vascular clinical features only, while the microscopic abnormal distribution of collagen fibers forming the microstructure of the vascular wall underlies a silent arteriopathy known as "weakness of the vessel wall", which is the real predisposing genetic risk for artery rupture and it does not present any clinical features or symptoms [2].

Ischemic and hemorrhagic vascular events, due to artery dissection or rupture, have been documented in these patients who usually claim headache, ipsilateral neck pain, incomplete Horner's syndrome (ptosis and myosis), bruits, transient ischemic attack, cerebrovascular accident signs such as amaurosis fugax,

\footnotetext{
* Corresponding author. Tel.: +39 055 7947112; fax: +39 0557947939.

E-mail address: mannelli.giuditta@gmail.com (G. Mannelli).
}

syncope, encephalopathy and focal signs [2,3]. Cervicocephalic arterial dissections and rupture could be traumatic or spontaneous, and they could follow mild efforts as head and neck maneuvering, forceful cough and vomiting [4]. Few authors report a syndrome called "spontaneous cervical arterial dissection (SCAD)", used to describe a nontraumatic tear or disruption in the wall of the brain-supplying arteries [5]

Here we report a female patient suffering from OI [6,7] who experienced a spontaneous rupture of internal carotid artery (ICA) during a neck dissection.

\section{Case report}

A 52-year-old female patient referred to our Institution for severe dysphonia and a left neck lump. Her medical history was positive for OI type I with several bone fractures and bleeding complications after adeno-tonsillectomy in 1980. The physical examination showed a right vocal fold paralysis, increased thyroid gland volume, and several bulky neck lymph nodes on both sides. One lymphadenopathy was $5 \mathrm{~cm} \times 6 \mathrm{~cm}$ in size and seemed to be adherent to the right cervical neurovascular bundle at level II. A preoperative CAT scan of the neck and chest showed multiple calcifications in neck lymph nodes (from level II to level VI) bilaterally and a thyroid gland mass with similar calcifications 

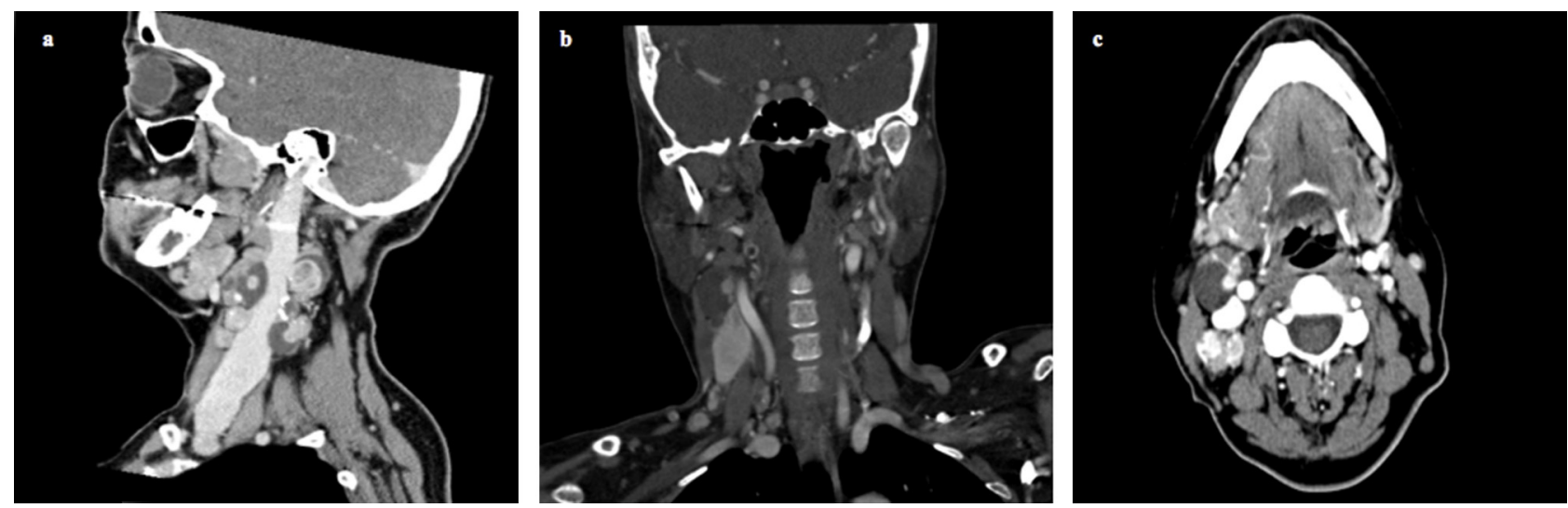

Fig. 1. TC scan imaging showing multiple cervical lymph node metastasis: (a) sagittal section, (b) coronal section and (c) axial section.

(Fig. 1). Vascular abnormalities were not evidenced. Subsequently, a fine needle aspiration biopsy was performed and a thyroid papillary carcinoma was found.

Thus, we performed total thyroidectomy with right recurrent nerve sacrifice and bilateral modified radical neck dissection including level VI.

During the right neck dissection, the cervical lymph node at sublevel IIA appeared to infiltrate the internal jugular vein (IJV) that was sacrificed (Fig. 2). During IJV ligation, manipulation of the vascular bundle determined a bleeding coming from a minimal leakage of ICA's wall which required its clamping. During these procedures we documented an evident retrograde blood flow from the Willi's Circle (Fig. 3). With the help of vascular surgeon, we removed the damaged tract of the ICA and he performed a transposition of the cranial stump of the ICA on the external carotid artery (ECA). The restoration of normal blood flow was intraoperatively confirmed by ultrasound echography. Finally, no cerebrovascular complications were found at the emergence from anesthesia.

The histopathologic evaluation confirmed a papillary carcinoma of the thyroid gland and bilateral neck metastases, pT4aN1b according to American Joint Committee on Cancer [8].

The histopathologic analysis of the damaged ICA tract did not show any cancer infiltrations of the artery's walls, while the signs of arterial wall dissection were documented (Fig. 4).

The patient was discharged 10 days later and she had not experienced any disease relapses after 12 months.

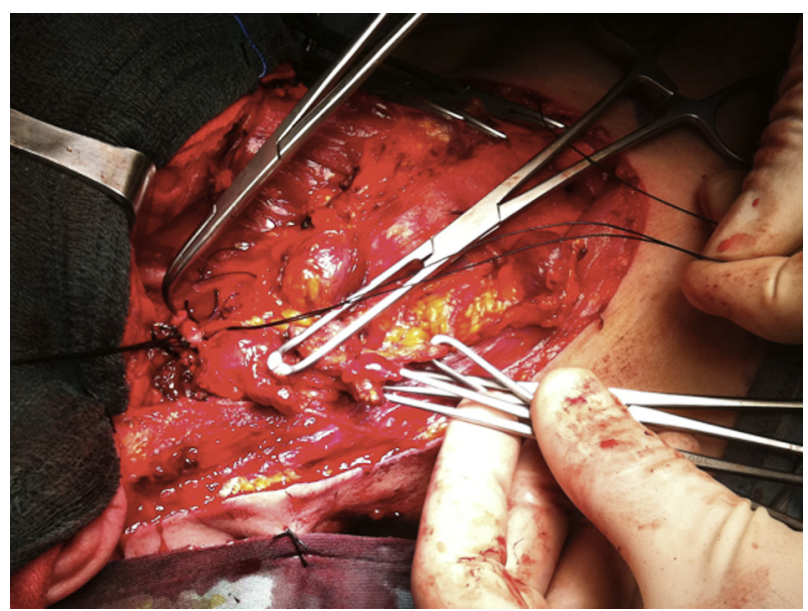

Fig. 2. Ligation of the right internal jugular vein (IJV) during neck dissection procedure: multiple and bulky lymph node metastases are visible in the surgical field. Two silky stitches have been placed around the cranial portion of the right IJV.

\section{Discussion}

Connective tissue disorders alter the structure of vascular walls, causing a silent arteriopathy known as "weakness of the vessel wall" [2], which does not present any clinical features and it is not diagnosed by common imaging techniques; dissection of carotid and vertebral arteries is reported as common feature in patients suffering from connective tissue disorders, but usually they claim alert symptoms. The most common locations of cervicocephalic artery anomalies are regions where these vessels are mobile, not firmly anchored to other arteries or bony structures. The ICA is relatively fixed proximally, at the origin from the common carotid artery, and distally, at the point of penetration in the petrous portion of the temporal bone. The arterial segment between these two points of anchorage is mobile and therefore vulnerable to stretching, especially during neck hyperextension with head rotation, which can stretch the ICA against an upper cervical vertebra or a prominent styloid process. The strain often occurs around $3 \mathrm{~cm}$ above the carotid bifurcation [5]. Usually, patients who experience cervical artery dissection or rupture might report either precipitating events or prior minor neck trauma [9], even if a clear cause-effect relationship is still debatable [10].

Our patient was affected by OI type I. Vascular complications are rare in OI when compared with other diseases involving connective tissue $[6,7]$. The most common vascular disorders are dysplasia of aortic and mitral valves, and congenital cardiac

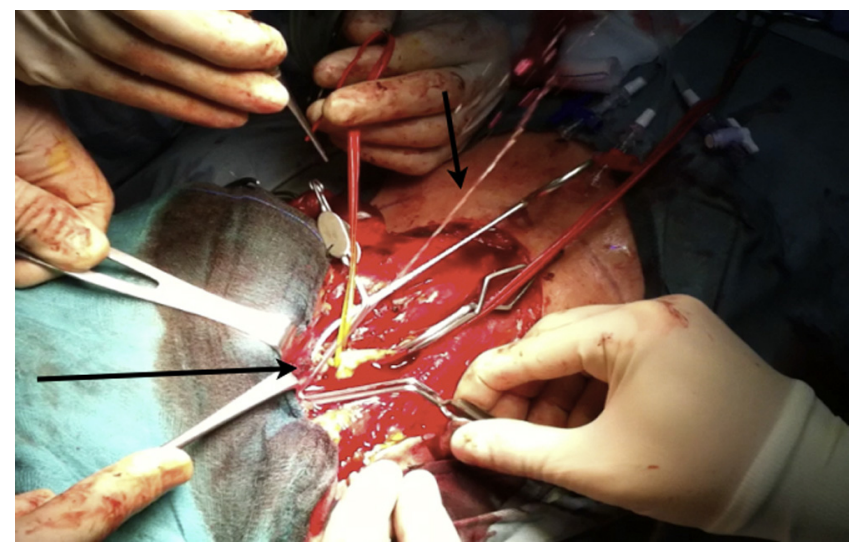

Fig. 3. Retrograde blood flow coming from the cranial resected stump of the internal carotid artery (ICA): arrows point the arterial blood jet coming from the cranial portion of the resected ICA. Red loops have been placed around the caudal stump of the ICA in order to perform the following anastomosis. (For interpretation of the references to color in this figure legend, the reader is referred to the web version of the article.) 


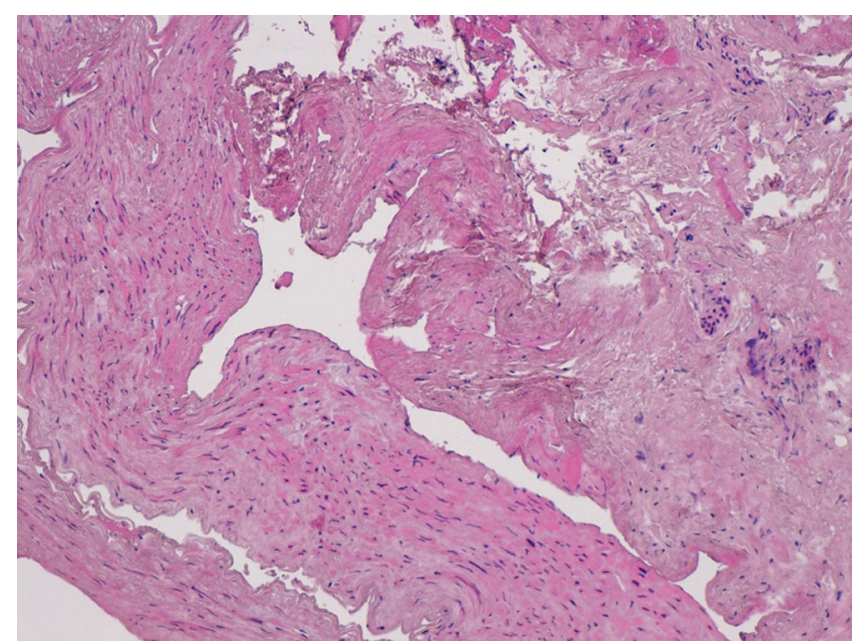

Fig. 4. ICA's wall alterations at optic microscopy: thickened intima and initial dissection at the adventitia level.

malformations. The ascending part of the aorta could be involved [11]. Spontaneous hemorrhages involving retina and conjunctivae, epistaxis and melena may occur due to capillary fragility. On the other hand, vascular cerebral complications are very rare [11]. Thus, the relationship between cervical artery events and OI is difficult to demonstrate [3].

Here we reported, to the best of our knowledge, the first case of spontaneous ICA's rupture that occurred during surgical neck dissection in a female patient suffering from OI with no evidence of vascular anomalies at the CAT scan. The rupture occurred during a gentle manipulation of this major vessel during a standard surgical procedure, without any direct trauma on it; thus, in our opinion, and in accordance with literature $[9,10,12]$, the intrinsic wall weakness was the predisposing cause of its rupture. Moreover, the fact that it was not a catastrophic artery rupture steers toward considering the connective tissue disorder as the main risk factor for this kind of potential life-threatening event, excluding improper surgical approaches and the absence of reported neoplastic vessel infiltration.

In accordance with our experience, we assert that surgical manipulation of the carotid bundle, which can occur during neck dissection procedures, should be performed carefully in patients with collagen disorders, because it could lead toward major vessel rupture and then to its sacrify, with potential severe clinical consequences. Additional imaging techniques such as contrastenhanced CAT scan, including angio-MRI, 3D-CTA, and carotid artery ultrasonography [1] might help in diagnosing the arteriopathy when evident, but the higher risk for spontaneous cervical artery rupture should be taken into consideration anyway.

\section{Conflict of interest}

All authors disclose no financial support or relationship that may pose a conflict of interest.

\section{Sources of support for the work}

No grants; no drugs; no special equipments.

\section{References}

[1] Lachman RS, Tiller GE, Graham Jr JM, Rimoin DL. Collagen, genes and the skeletal dysplasias on the edge of a new era: a review and update. Eur J Radiol 1992:14:1-10.

[2] Brand T, Orberk E, Weber R, Werner I, Busse O, Müller BT, et al. Pathogenesis of cervical artery dissections: association with connective tissue abnormalities. Neurology 2001;57:24-30.

[3] Schievink WI, Mokri B. Familial aorto-cervicocephalic arterial dissections and congenitally bicuspidic aortic valve. Stroke 1995;26:1935-40.

[4] Kumar SD, Kumar V, Kaye W. Bilateral internal carotid artery dissection from vomiting. Am J Emerg Med 1998;16:669-70.

[5] Campos-Herrera CR, Scaff M, Yamamoto FI, Conforto AB. Spontaneous cervical artery dissection: an update on clinical and diagnostic aspects. Arq Neuropsiquiatr 2008;66:922-7.

[6] Forlino A, Marini JC. Osteogenesis imperfecta: prospects for molecular therapeutics. Mol Genet Metab 2000;71:225-32.

[7] Rouvière S, Michelini R, Sarda P, Pagès M. Spontaneous carotid artery dissection in two siblings with osteogenesis imperfecta. Cerebrovasc Dis 2004;17: $270-2$.

[8] Baldridge D, Schwarze U, Morello R, Lennington J, Bertin TK, Pace JM, et al. CRTAP and LEPRE1 mutations in recessive osteogenesis imperfecta. Hum Mutat 2008;29:1435-42.

[9] Lee VH, Brown Jr RD, Mandrekar JN, Mokri B. Incidence and outcome of cervical artery dissection: a population-based study. Neurology 2006;67: 1809-12;

Bogousslavsky J, Pierre P. Ischaemic stroke in patients under age 45. Neurol Clin 1992;10:113-24.

[10] Rothwell DM, Bondy SJ, Williams JL. Chiropractic manipulation and stroke: a population-based case-control study. Stroke 2001;32:1054-60.

[11] Isotalo PA, Guindi MM, Bedard P, Brais MP, Veinot JP. Aortic dissection: a rare complication of osteogenesis imperfecta. Can J Cardiol 1999;15:1139-42.

[12] Schievink WI, Michels VV, Piepgras DG. Neurovascular manifestations of heritable connective tissue disorders. Stroke 1994;25:889-903. 